\title{
Spatial distribution of bacteria associated with the marine sponge Tethya californiana
}

\author{
Detmer Sipkema $\cdot$ Harvey W. Blanch
}

Received: 17 March 2009/Accepted: 11 November 2009/Published online: 3 December 2009

(C) The Author(s) 2009. This article is published with open access at Springerlink.com

\begin{abstract}
Microbial diversity and spatial distribution of the diversity within tissue of the marine sponge Tethya californiana was analyzed based on 16S rRNA gene sequences. One candidate division and nine bacterial phyla were detected, including members of all five subdivisions of Proteobacteria. Moreover, chloroplast-derived Stramenopiles- and Rhodophyta-affiliated 16S rRNA gene sequences were found and Stramenopiles represented the most abundant clones (30\%) in the clone library. On the phylum-level, the microbial fingerprint of $T$. californiana showed a similar pattern as its Mediterranean relative T. aurantium. An interesting difference was that Cyanobacteria that were abundantly present in $T$. aurantium were not found in $T$. californiana, but that the latter sponges harbored phototrophic Stramenopiles instead. Surprisingly, the phototrophic microorganisms were evenly distributed over the inner and outer parts of the sponge tissue, which implies that they also reside in regions without direct light exposure. The other phyla were also present in both the outer cortex and the mesohyl of the sponges. These results were confirmed by analysis on the operational taxonomic unit level. This leads to the conclusion that from a qualitative point of view, spatial distribution of microorganisms in T. californiana tissue is quite homogeneous. Thirty-two percent of the operational taxonomic units shared less than $95 \%$ similarity with any other known sequence. This indicates that marine sponges are a rich source of previously undetected microbial life.
\end{abstract}

Communicated by M. Kühl.

D. Sipkema $(\bowtie) \cdot H$. W. Blanch

Department of Chemical Engineering,

University of California Berkeley,

201 Gilman Hall, Berkeley, CA 94720, USA

e-mail: detmer.sipkema@wur.nl

\section{Introduction}

Marine sponges are reservoirs of many unknown, and certainly of many uncultured, microbial species. The microbial inhabitants of a growing number of sponge species have been characterized with molecular tools (Taylor et al. 2007 and references cited therein), and it has been suggested that sponges harbor many specific bacterial species and clades that are not found in other environments (Hentschel et al. 2002). Initially, these sponge-specific clades could still be the result of insufficient sampling, but huge sequencing efforts during the last decade have supported this hypothesis. However, very little information exists about the actual relationship between the host and its specifically associated bacteria and the distribution of these bacteria throughout the sponge. Although many potential beneficial roles have been ascribed to the microorganisms, it has been difficult to find in situ evidence for these hypotheses due to the complexity of the host-bacteria network. For that reason, scientists have been hesitant to call the associated bacteria 'symbionts'. However, sponges are considered to be the oldest metazoans still present, and their presumably consistent association with microorganism through time and space strongly suggests a functional relationship between the sponge and its bacteria. Sponges provide a niche that is richer in nutrients than seawater and sediments (reviewed in Lee et al. 2001) and could prevent the inhabitants from drifting away to less favorable environments. In addition, the primary and secondary metabolism of the microorganisms and the host may be interrelated in such a way that bacteria utilize sponge's metabolic waste products (ammonia, nitrate, carbon dioxide), while sponge cells could potentially make use of, e.g., oxygen and small organic molecules excreted by associated phototrophic microbes (Wilkinson and Garrone 1980; 
Hallam et al. 2006; Bayer et al. 2008; Hoffmann et al. 2009). Moreover, it has become clear that bioactive secondary metabolites produced by associated microbes play an important role in reducing predation (Faulkner 2000). If associated bacteria are involved in the chemical defense of the host, their preferable location in the sponge could be near the surface, which is most vulnerable to predation and larval settlement. A substantial number of bacteria isolated from the surface of marine sponges have indeed shown to produce bioactive compounds (Becerro et al. 1994; Osinga et al. 2001; Chelossi et al. 2004; Müller et al. 2004). In addition, photosynthetically active cyanobacteria and microalgae are also generally found in the outer tissue layer of the sponge (reviewed in Hentschel et al. 2003), but exceptions to this general picture are not uncommon. For example, the cyanobacteria Oscillatoria spongeliae was found abundantly in the mesohyl of Lendenfeldia chondrodes but not in the epithelial sections (Ridley et al. 2005). The inner core of sponges is generally populated by heterotrophic and autotrophic bacteria (reviewed in Hentschel et al. 2003), and it has been shown in times of ceased pumping activity, which frequently occurs for many sponges, this inner core can easily become anoxic (Hoffmann et al. 2005, 2008), creating a perfect niche for facultatively anaerobic species.

A clear idea of spatial distribution of associated bacteria is important to understand the relationship between the sponge and its associated microorganisms, and currently very little detailed information exists on this topic. However, a few studies have indicated that different microbial profiles are likely to exist between exterior and interior tissue samples (Wichels et al. 2006; Thiel et al. 2007). Knowledge of the location of associated species in the sponge may provide information about the preferred microenvironment of a particular strain, which can be used to design cultivation experiments as these organisms are still regarded as mostly uncultivable (e.g., Friedrich et al. 2001).

We have studied the spatial variation of associated microorganisms of Tethya californiana (De Laubenfels, 1932). This is an ideal species for this type of studies as it has a clearly defined mesohyl and cortex (Fig. 1). In addition, the sponge is ball-shaped, which results in the largest possible distance from the core of the sponge to the surface and increases the chance of occurrence of gradients in the tissue.

\section{Materials and methods}

Sample collection

Two Tethya californiana specimens (Tc1 and Tc2) were collected near Monastery Beach (Monterey, CA, USA) at a

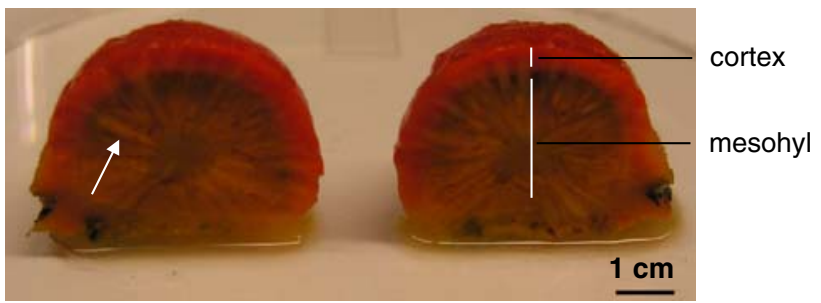

Fig. 1 A Tethya californiana specimen cut into halves. The orange outer tissue can easily be distinguished from the inner brownish core. In addition, long whitish spicules can be observed that are positioned radially from the core to the periphery (see e.g., white arrow)

depth of approximately $8 \mathrm{~m}$ on May 24, 2006. They were immediately transported in excess of natural seawater to the laboratory and processed within $2 \mathrm{~h}$. The sponges were rinsed 3 times with sterile artificial seawater (natural sea salt mix, Oceanic Systems, Dallas, TX, USA) before preparing cortex and mesohyl samples. Special care was taken to avoid contact between the two tissue types and a safety margin in the tissue between mesohyl and cortex was used when the samples were cut. The samples were separately ground with a sterilized mortar and pestle. Two tissue volumes of sterile artificial seawater (ASW) were added to the tissue samples to obtain homogeneous cell suspensions. Each cell suspension was divided in aliquots of $1.2 \mathrm{ml}$ and mixed with $0.6 \mathrm{ml} 50 \%$ glycerol in ASW. The samples were frozen until $-20^{\circ} \mathrm{C}$ before they were stored at $-80^{\circ} \mathrm{C}$.

\section{DNA library construction}

Genomic DNA was extracted from the cryopreserved cells using the Puregene DNA isolation kit (Gentra, Minneapolis, MN, USA). Cryopreserved cell suspension with the volume of $400 \mu \mathrm{l}$ was used for DNA isolation. The cell suspension was centrifuged, and the supernatant was decanted. DNA was isolated according to manufacturer's specifications.

Universal eubacterial primers $8 \mathrm{~F}$ (5'-AGA GTT TGA TCC TGG CTC AG-3' ${ }^{\prime}$ [Felske et al. 1997]) and 1492R (5'GGT TAC CTT GTT ACG ACT T-3' [Lane 1991]) were used to amplify 16S rRNA gene fragments. The PCR conditions were as follows: initial denaturation for $10 \mathrm{~min}$ at $95^{\circ} \mathrm{C} ; 30$ cycles of denaturation for $30 \mathrm{~s}$ at $95^{\circ} \mathrm{C}$, annealing for $30 \mathrm{~s}$ at $55^{\circ} \mathrm{C}$ and elongation for $1 \mathrm{~min} 45 \mathrm{~s}$ at $72^{\circ} \mathrm{C}$, and a final extension step for $10 \mathrm{~min}$ at $72^{\circ} \mathrm{C}$.

PCR Products of successful reactions were cloned with a TOPO TA cloning kit for sequencing (Invitrogen, Carlsbad, CA, USA) in accordance with the manufacturer's instructions. A total of 384 colonies were picked, and plasmids were purified for each specimen (192 for the cortex and 192 for the mesohyl).

Plasmids containing a $16 \mathrm{~S}$ rRNA gene PCR product were sequenced using the $8 \mathrm{~F}$ primer. Sequence chromatograms 
were manually inspected and assembled using the Contig Express software (Invitrogen). Sequences with similarities $>99 \%$ were considered as one operational taxonomic unit (OTU) (Stackebrandt and Ebers 2006). In addition, they were checked for possible chimerical origins by using the program Bellerophon (version 3) from the Greengenes website (Huber et al. 2004). Sequences were deposited in Genbank under accession numbers: EU290173-EU290248, EU290250-EU290266, EU290268-EU290275, EU290277EU290401 and EU290403-EU290460.

\section{Phylogenetic analysis}

The DNA sequences of the OTUs and nearest neighbors (as determined using the greengenes database http:// greengenes.lbl.gov) were imported in the ARB software package (Ludwig et al. 2004). DNA sequences were aligned using the FastAlign function of the alignment editor implemented in the ARB program and refined manually. Ambiguous regions of the alignment were systematically removed using the program Gblocks v.0.91b (Castresana 2000). The default program parameters were used, exclusive of allowing a minimum block length of 5 and gaps in $50 \%$ of positions. Bayesian (MB), maximum likelihood (ML) and maximum parsimony (MP) phylogenetic methods were employed to estimate phylogenetic relationships in the data sets. MP trees were generated with PAUP* (Swofford 2003). Heuristic searches consisted of 1000 random stepwise-addition-sequence replicates using TBR branch swapping. ML trees were calculated with the GTR model and estimated proportion of invariable sites and gamma distribution in GARLI v0.951 (Zwickl 2006). Runs were automatically terminated when tree topology did not change for 10,000 generations. Five separate runs were performed, and the tree with the best likelihood was selected. Bayesian analyses were performed using MrBayes v3.0b4 (Huelsenbeck and Ronquist 2001) at the freely available Bioportal server (www.bioportal.uio.no). All parameters were treated as unknown variables with uniform prior-probability densities at the beginning of each run, and their values were estimated from the data during the analysis (Ronquist et al. 2005). All Bayesian analyses were initiated with random starting trees and were run for $1 \times 10^{7}$ generations.

\section{Rarefaction analysis}

The extent of diversity examined in the clone libraries was determined by rarefaction analysis. Rarefaction curves were obtained using the algorithm described by Hurlbert (1971) with the freeware program aRarefactWin (http:// www.uga.edu/ strata/software.html). Rarefaction curves were plotted, and regressions were calculated using two different regression equations (Thiel et al. 2007): $y=a\left(1-e^{-b x}\right)$

$y=a\left(1-e^{-b x^{\wedge} c}\right)$

where $x$ is the sample size, $y$ the observed number of OTUs, $a$ the expected number of OTUs with infinite sample size, and $b$ and $c$ are regression variables.

Statistical analysis

Mesohyl and cortex samples were compared for the presence or absence of OTUs that were present in at least two samples with the PRIMER 6 Software (Clarke and Warwick 2001). The qualitative data were used to calculate BrayCurtis similarity to evaluate the importance of two factors: tissue type and specimen. Nonmetric multidimensional scaling (MDS) was used to plot the analysis. Based on the Bray-Curtis similarity index, in MDS plots, the distance between points is proportional to their degree of similarity; so, closer points are more similar than points farther away.

\section{Results and discussion}

Phylum-level analysis of $T$. californiana-associated microorganisms

A total of 680 high-quality partial $16 \mathrm{~S}$ rRNA gene clone sequences (average $\sim 900 \mathrm{bp}$ ) were obtained that were quite evenly distributed over the cortex and mesohyl samples of the two Tethya californiana specimens (179 for T1cor, 155 for T1mes, 166 for T2cor and 180 for T2mes). Representatives of nine bacterial phyla including the subdivisions $\alpha$-, $\beta$-, $\gamma$-, $\delta$ - and $\varepsilon$-Proteobacteria and one candidate division were present in $T$. californiana (we did not screen for Archaea). In addition, phototrophic Stramenopiles- and Rhodophyta-affiliated sequences were present in the clone libraries (Fig. 2a-e). Clones containing sequences related to Stramenopiles-derived chloroplasts were actually the most abundant ones in the combined clone libraries (30\%). Other major groups were $\alpha$-Proteobacteria (23\%), $\gamma$-Proteobacteria (15\%), Bacteroidetes $(9 \%)$ and $\beta$ Proteobacteria (8\%), while $\delta$-Proteobacteria, Firmicutes and Verrucomicrobia (all $\sim 3 \%$ ) were represented by lower numbers of clones (Fig. 2a). Actinobacteria (0.7\%) and $\varepsilon-$ Proteobacteria $(0.3 \%)$ were present in very low numbers and were only detected in T1 (Fig. 2b, c), while Rhodophyta $(0.3 \%)$ were only detected in T2 (Fig. 2d, e). Planctomycete clones were more abundantly obtained from mesohyl samples $(3.4 \pm 2.5 \%)$ than from cortex samples $(0.6 \pm 0.03 \%)$, but in general, the distribution of clones over the microbial phyla was roughly comparable for both specimens and both locations within the specimens. 
Fig. 2 a-e Distribution of $16 \mathrm{~S}$ rRNA gene PCR products over the bacterial phyla and Stramenopiles and Rhodophyta for separate cortex and mesohyl samples from two $T$.

californiana specimens and for the total of the clones. The numbers of clones are in parentheses. The phyla as listed in the legend are depicted clockwise starting at $12 \mathrm{O}^{\prime}$ clock. Acidobacteria is only present in T1mes, T2cor and T ALL. Actinobacteria, \&Proteobacteria and Nitrospirae are only present in $\mathrm{T} 1$ and $\mathrm{T}$ ALL. Rhodophyta was found only in T2cor
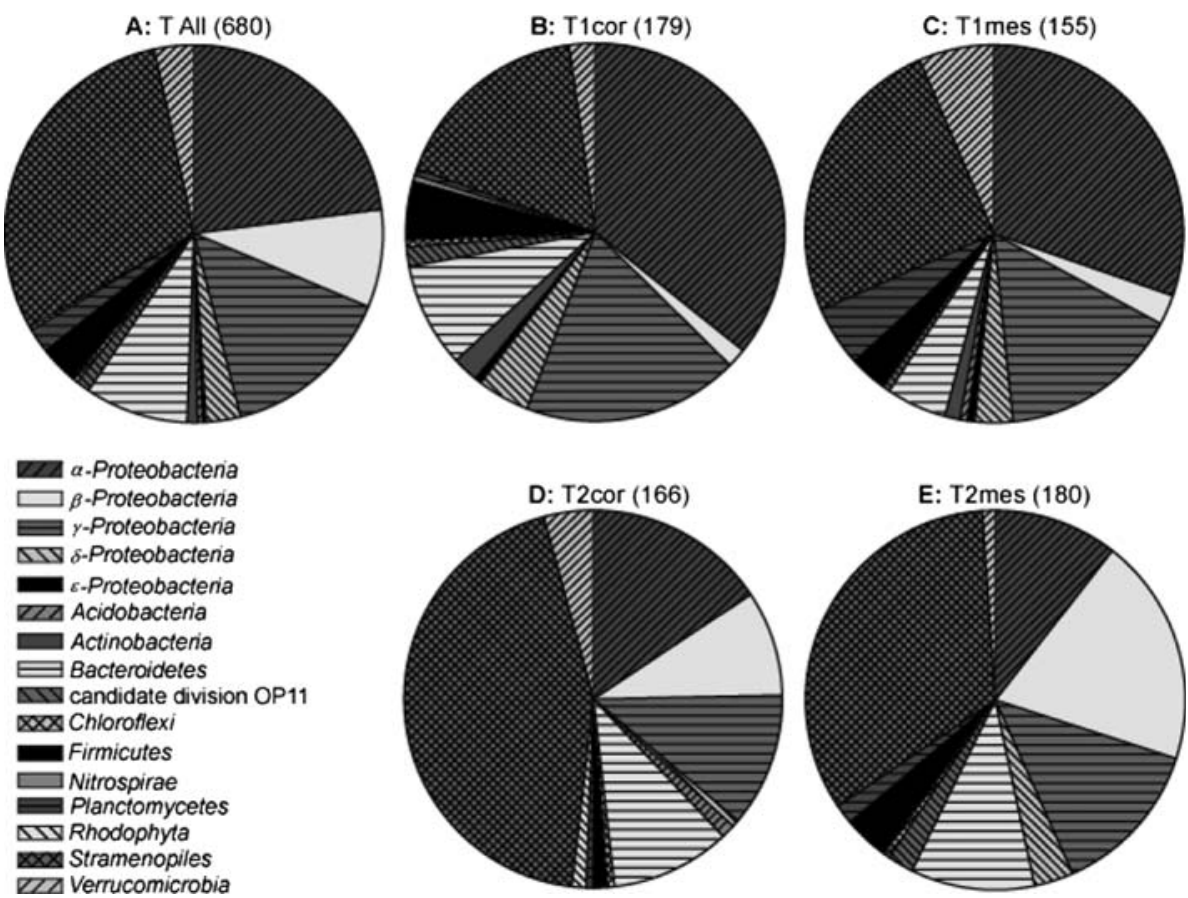

The major phylogenetic distribution of T. californiana $16 \mathrm{~S}$ rRNA gene clones is comparable to the distribution in Tethya aurantium (Thiel et al. 2007). The bulk of $16 \mathrm{~S}$ rRNA gene clone sequences from $T$. aurantium was derived from Proteobacteria (38\%), followed by Cyanobacteria $(27 \%)$ and Bacteroidetes (25\%). We did not find any cyanobacterial sequence, but phototrophic Stramenopile chloroplast-affiliated sequences represented $30 \%$ of all clones. Phototrophic organisms (green algae) associated with the sponges Ephydatia fluviatilis and Haliclona cymiformis translocate metabolites to their hosts (Wilkinson 1980; Grant et al. 2006), and it could be speculated that the presence of Cyanobacteria in T. aurantium and phototrophic Stramenopiles in T. californiana are different solutions with a similar outcome with respect to interactions in the sponge-microbe network. A difference between T. californiana and T. aurantium is that for the latter sponge phototrophic associates were mostly confined to the mesohyl (Thiel et al. 2007). Microbial profiles of other, more distantly related, sponges are considerably different from the Tethya species, especially with respect to the fraction of phototrophic microorganisms (Hill et al. 2006).

The preferred habitat of the T. californiana, on top of exposed rocks in relatively shallow water $(5-10 \mathrm{~m})$, guarantees the availability of sufficient light for its phototrophic microorganisms in the cortex. However, members of the genus Tethya also harbor phototrophic microorganisms in their interior: Cyanobacteria in T. aurantium (Thiel et al. 2007) and green alga in T. seychellinsis (Gaino and Sará 1994). It has recently been shown that the thick radially positioned spicules conduct enough light into the core of Tethya aurantium to sustain the growth of phototrophic microorganisms (Brümmer et al. 2008). Thus, phototrophic Stramenopiles could obtain their energy from light inside T. californiana.

Estimation of microbial diversity

Based on the rule of thumb of Stackebrandt and Ebers (2006) that sequences that share less than 99\% similarity are considered to be derived from a different operational taxonomic unit (OTU), the 680 clones represented 263 different OTUs. Rarefaction curves showed that the actual diversity is probably well over 400 OTUs for T. californiana and that especially for the cortex samples diversity was not completely covered (Table 1). However, the high number of analyzed clones suggests that the part of bacterial diversity that was not detected probably represents a minor quantitative fraction in the sponge. The number of detected OTUs was higher in the cortex samples (101 and 87 for $\mathrm{Tc} 1$ and $\mathrm{Tc} 2$, respectively) than in the mesohyl samples (77 and 62 for $\mathrm{Tc} 1$ and $\mathrm{Tc} 2$, respectively). In addition, the number of detected OTUs in Tc1 was slightly higher than in Tc2.

A similar general pattern for diversity in cortex and mesohyl was observed by Thiel et al. (2007) for the Mediterranean sponge Tethya aurantium. The number of OTUs detected in $T$. californiana (cortex $92.5 \pm 9.2$; mesohyl $67 \pm 8.5$ OTUs) was significantly higher than in T. aurantium tissue (cortex $16 \pm 14.1$; mesohyl $11 \pm 2.8$ OTUs), but this is probably caused by a lower clone 
Table 1 Observed and expected bacterial diversity in Tethya californiana

\begin{tabular}{|c|c|c|c|c|c|c|}
\hline \multirow[t]{2}{*}{ Clone library } & \multirow[t]{2}{*}{$n$} & \multirow[t]{2}{*}{ OTU } & \multicolumn{2}{|c|}{ Regression (1) } & \multicolumn{2}{|c|}{ Regression (2) } \\
\hline & & & $a$ & Coverage $(\%)$ & $a$ & Coverage $(\%)$ \\
\hline Tclect & 179 & 101 & 170 & 60 & 179 & 56 \\
\hline Tc1mes & 155 & 77 & 115 & 67 & 142 & 54 \\
\hline Tc2ect & 166 & 87 & 145 & 60 & 200 & 43 \\
\hline Tc2mes & 180 & 62 & 76 & 81 & 82 & 76 \\
\hline Tc all & 680 & 263 & 405 & 65 & 496 & 53 \\
\hline
\end{tabular}

$a$ is the expected number of OTUs according to regression models 1 and 2 of the rarefaction curves

The coverage is calculated by dividing the number of observed OTUs by the expected number of OTUs: OTU $/ a \times 100 \%$

All $R^{2}$ of the regression models and the rarefaction points were larger than 0.99

number per sample for the latter sponge (on average: $32.8 \pm 3.3$ clones).

OTU-level analysis of $T$. californiana-associated microorganisms

In order to obtain more detailed insight in the microbial populations associated with $T$. californiana, phylogenetic trees containing the detected OTUs and their nearest neighbors were constructed (Figs. 3, 4, 5, 6). Many near neighbors were derived from other sponges, but only a few OTUs from $T$. californiana seem to be really sponge-specific, because for many OTUs sponge-derived neighbors (if present) were accompanied by nonsponge-derived neighbors. In order to bring to light microorganisms that were potentially derived from the surrounding seawater, we compared the sponge-derived sequences with 94 16S rRNA gene sequences that were obtained from Monterey Bay bacterioplankton samples in other studies (data not shown: e.g., Suzuki et al. 2001, 2004). This analysis revealed that four $T$. californiana-derived OTUs (OTU08Tcor, OTU10T1, OTU79Tmes and T1cor2D6) probably originated from seawater (Figs. 3, 5).

Among the $\alpha$-Proteobacteria-affiliated OTUs, one OTU, which was retrieved from both cortex and mesohyl from Tc1 (OTU15T1), seems to be sponge- or even Tethyaspecific (Fig. 3). It is only distantly related to other known $\alpha$-Proteobacteria. Its nearest neighbor is a clone that was obtained from a $T$. aurantium cortex sample (97\% identity), and it shares less than $90 \%$ identity with any other sequence in Genbank. The most abundant $\alpha$-Proteobacteria-affiliated clone sequences OTU17T1 (6.3\% of all clone sequences) and OTU47T2 (3.7\%) were present in both mesohyl and cortex of Tethya1 and Tethya2, respectively.

Only one $\beta$-Proteobacteria-affiliated OTU (OTU53T) was present in $T$. californiana. It was detected in both cortex and mesohyl samples of both specimens. Based on phylogenetic analysis, the OTU belongs to a clade with only sponge-derived sequences (Fig. 4). Its nearest neighbor is an isolate from the Pacific sponge Haliclona (?gellius) sp., and other related sequences were obtained from sponges from a wide range of geographical regions. For the Tethya aurantium-derived neighbors, it was found that clone sequences in this cluster represented 19\% of all clones sequences (Thiel et al. 2007) and also for T. californiana they represented a large fraction $(8.7 \%)$ of all clone sequences. The fact that it was isolated from all tissue samples implies that this bacterial species is potentially an important partner for sponges, which role still needs to be identified.

A wide variety of $\gamma$-Proteobacteria-affiliated OTUs were found in $T$. californiana. OTU60T was the most abundant $\gamma$-proteobacterial sequence in the clone library with $3.5 \%$ of all clones and was detected in all mesohyl and cortex samples. Although it is distantly related to an Aplysina aerophoba clone, nearest neighbors were derived from nonsponge sources (Fig. 4). With two exceptions, no sponge-specific $\gamma$-Proteobacteria were detected in T. californiana. In addition, only for OTU60T and OTU78Tmes, overlap between sponge specimens was found. This indicates that most associated $\gamma$-Proteobacteria are most likely rather temporary dwellers than permanently associated microbes with $T$. californiana. Only T2mes2D12 and T1mes2G2 grouped in a cluster with sponge-derived clones and isolates. In the sponge Haliclona simulans, clones belonging to the latter cluster represented $44 \%$ of the total sponge library (Kennedy et al. 2008). However, both $\gamma$ proteobacterial clones belonging to putative sponge-related clusters were detected only as a single clone in the mesohyl sample of one of the specimens.

Only a few $T$. californiana Bacteroidetes-affiliated sequences have sponge-derived nearest neighbors (Fig. 5) and most of their sponge-derived neighbors were obtained from Haliclona (?gellius) sp., a species that is found in the vicinity of $T$. californiana (Sipkema et al. 2009), which could indicate that they are related to the environment rather than specifically to sponges. Despite the presence of significant numbers of Bacteroidetes-affiliated clone sequences in T. aurantium (25\%) (Thiel et al. 2007) and $T$. californiana (9\%), no closely related OTUs were observed for the two Tethya species. This is in agreement with the observation that sponge-specific clusters are not prevalent for Bacteroidetes (Taylor et al. 2007). OTU28T was the most abundant Bacteroidetes-affiliated sequence in $T$. californiana and represented $12 \%$ of Bacteroidetes-affiliated clones (1\% of all clones) and was present in both cortex and mesohyl samples from the two T. californiana specimens. Its nearest neighbors were isolated from different environments, and it probably represents a cosmopolitan bacterium. 


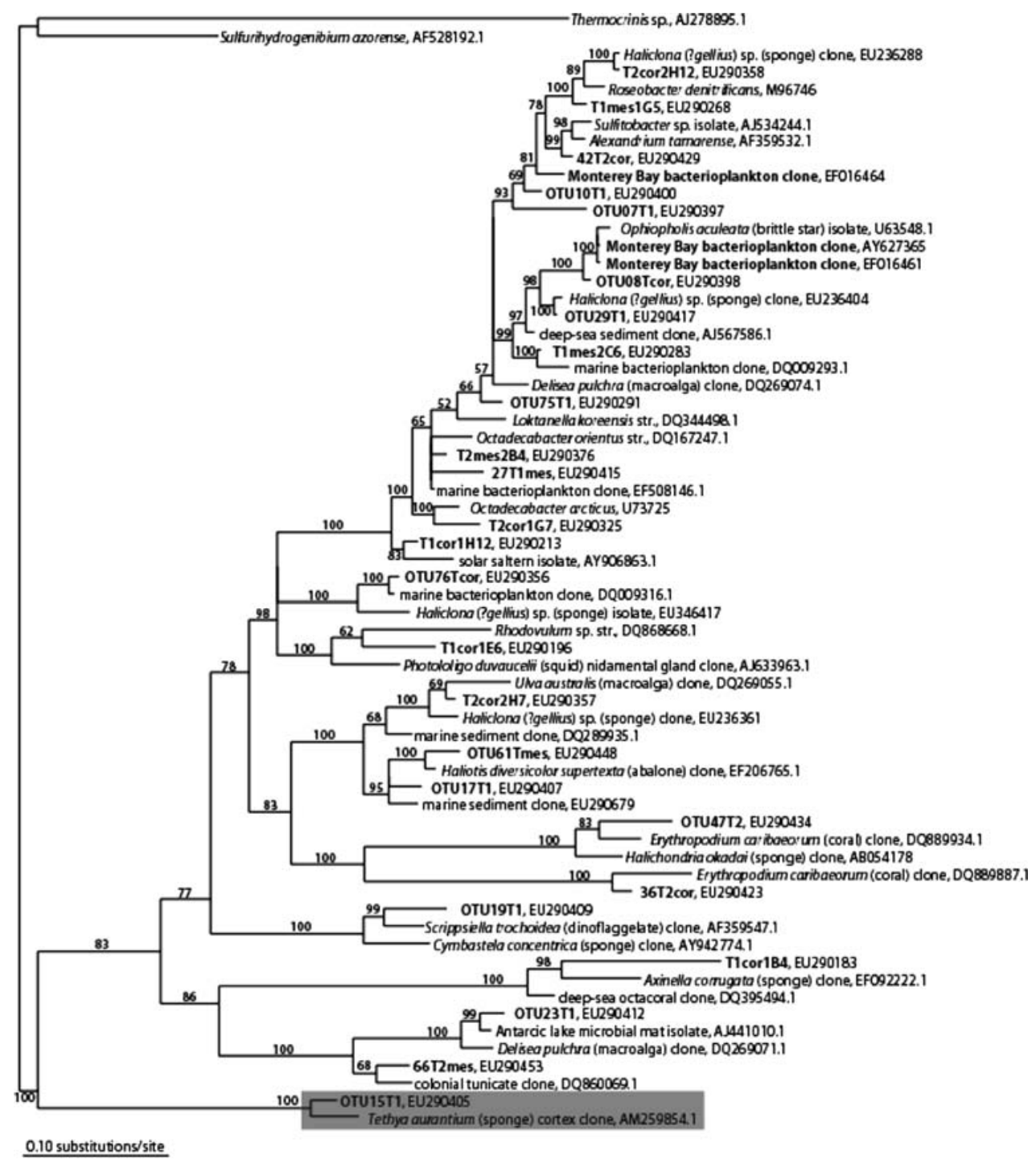

Fig. 3 Bayesian phylogram of $\alpha$-Proteobacteria 16S rRNA gene sequences from $T$. californiana bacteria (in bold) and their nearest neighbors (greengenes database August 18, 2007). The numbers above or below the branches correspond to posterior probability (PP) values of the Baysian analysis. OTUs that were found in more than one sample start with the letters 'OTU' followed by a code indicating the samples in which it was detected: $\mathrm{T} 1=T$. californiana specimen $1, \mathrm{~T} 2=T$. californiana specimen $2, \mathrm{~T}=$ both specimens, cor $=$ cortex, mes = mesohyl, no addition of 'cor' or 'mes' = found in both locations. OTUs that were found multiple times in only one individual

Stramenopiles chloroplast-affiliated OTUs were among the most abundantly obtained clone sequences. They represent closely related phylotypes that are not confined to sponges but are also detected in phytoplankton and soil samples (Fig. 6a). This is consistent with the finding that all photosynthetic Stramenopiles are monophyletic based on 18S rDNA sequences (Massana et al., 2004). Although very few microbial eukaryote sequences have been isolated from marine sponges (Taylor et al. 2007), photosynthetic Stramenopile-affiliated clones were also abundant and are encoded with a number followed by the individual and location where it was found. If $T$. californiana OTUs were more closely related to each other than to any other sequence, only one of the OTUs was used to construct the tree. The gray box indicates a Tethyaspecific cluster. Thermocrinis sp. and Sulfurihydrogenibium azorense were used as outgroup. Seawater-derived sequences obtained from Monterey Bay (the sampling site) are in bold. Phylogenetic tree topologies generated with a parsimony and maximum likelihood model were highly similar (data not shown)

stably associated with the sponge Cymbastela concentrica (Taylor et al. 2004).

For the other phyla, no sponge-specific clusters were observed (Fig. 6a, b), except for perhaps one Acidobacterium (T2cor2F4). This clone sequence is related to Acidobacteria derived from the sponges Mycale laxissima and Axinella corrugata and has no other near relatives. However, the clone was obtained as a singleton from only one tissue sample and is, therefore, probably only a minor constituent of the $T$. californiana microflora. OTUs 
Fig. 4 Bayesian phylogram of $\beta$ - and $\gamma$-Proteobacteria 16S rRNA gene sequences from $T$. californiana bacteria (in bold) and their nearest neighbors (greengenes database August 18, 2007). The gray boxes indicate putative spongespecific clusters. For further explanation, see Fig. 3

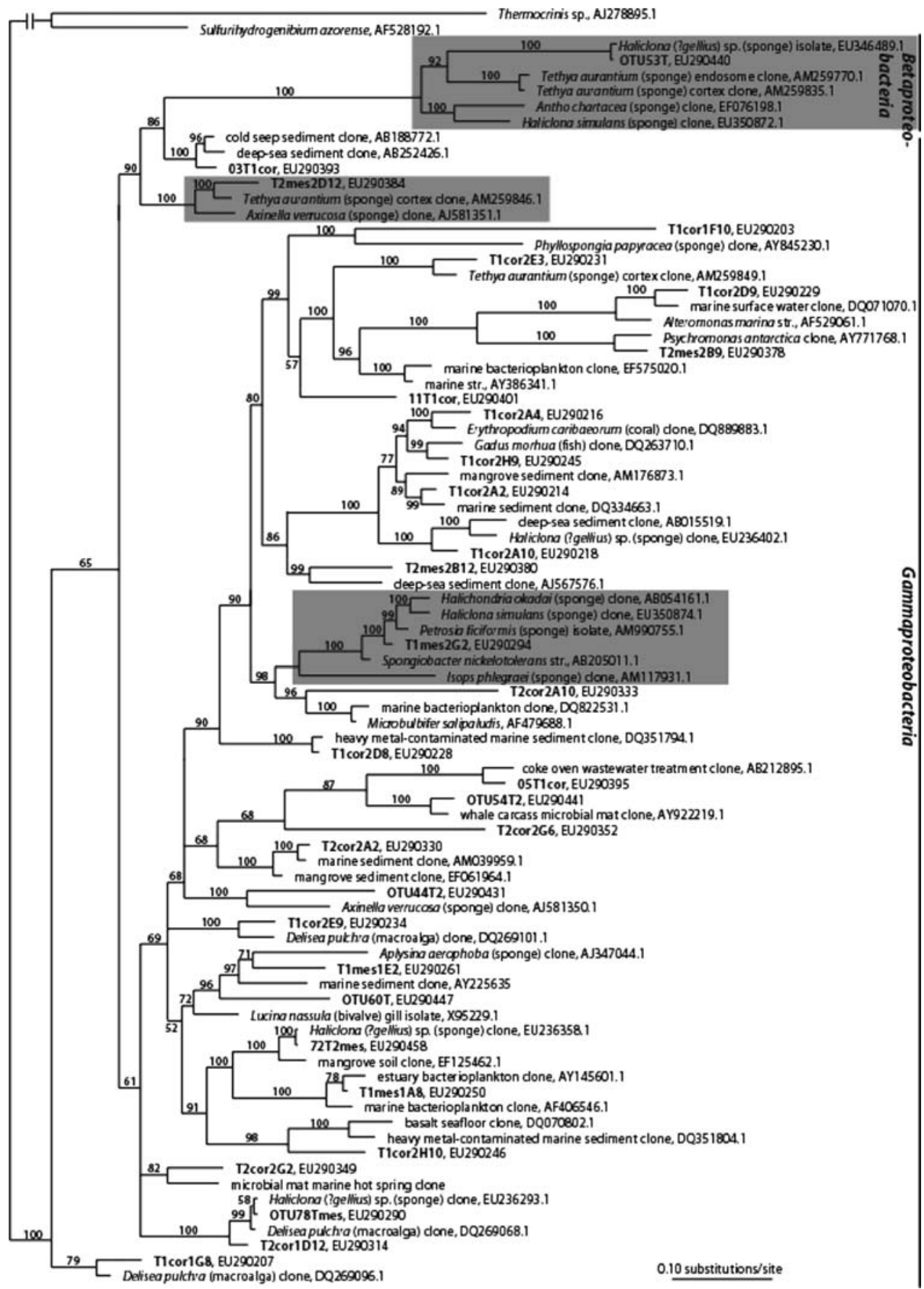

affiliated to Chloroflexi, Nitrospirae, Actinobacteria, $\delta$ Proteobacteria, \&-Proteobacteria, candidate division OP11, Verrucomicrobia, Planctomycetes and Firmicutes represented only a small fraction of the clone library. The only exception is Firmicutes-affiliated OTU57T that was present in both cortex and mesohyl samples from the two $T$. californiana specimens and represented $2.8 \%$ of all clones. Firmicutes quite commonly represent a significant percentage of bacterial 16S rRNA gene clone sequences from polar and cold-temperate marine sponges (Hill et al. 2006); however, sponge-specific clusters are uncommon (Taylor et al. 2007).

A remarkably high fraction of the $T$. californianaderived OTUs shared less than $95 \%$ with any other sequence in Genbank. Twenty percent of all sequences in the $16 \mathrm{~S}$ rRNA gene clone library (32\% of all OTUs) belong to this category (BLAST search April 29, 2008). Although most of these OTUs were detected in only one tissue sample and are unlikely to be sponge-specific or important partners for the sponge, they demonstrate the aptness of 
Fig. 5 Bayesian phylogram of Bacteroidetes 16S rRNA gene sequences from $T$. californiana bacteria (in bold) and their nearest neighbors (greengenes database August 18, 2007). Seawater-derived sequences obtained from Monterey Bay (the sampling site) are also in bold. For further explanation, see Fig. 3

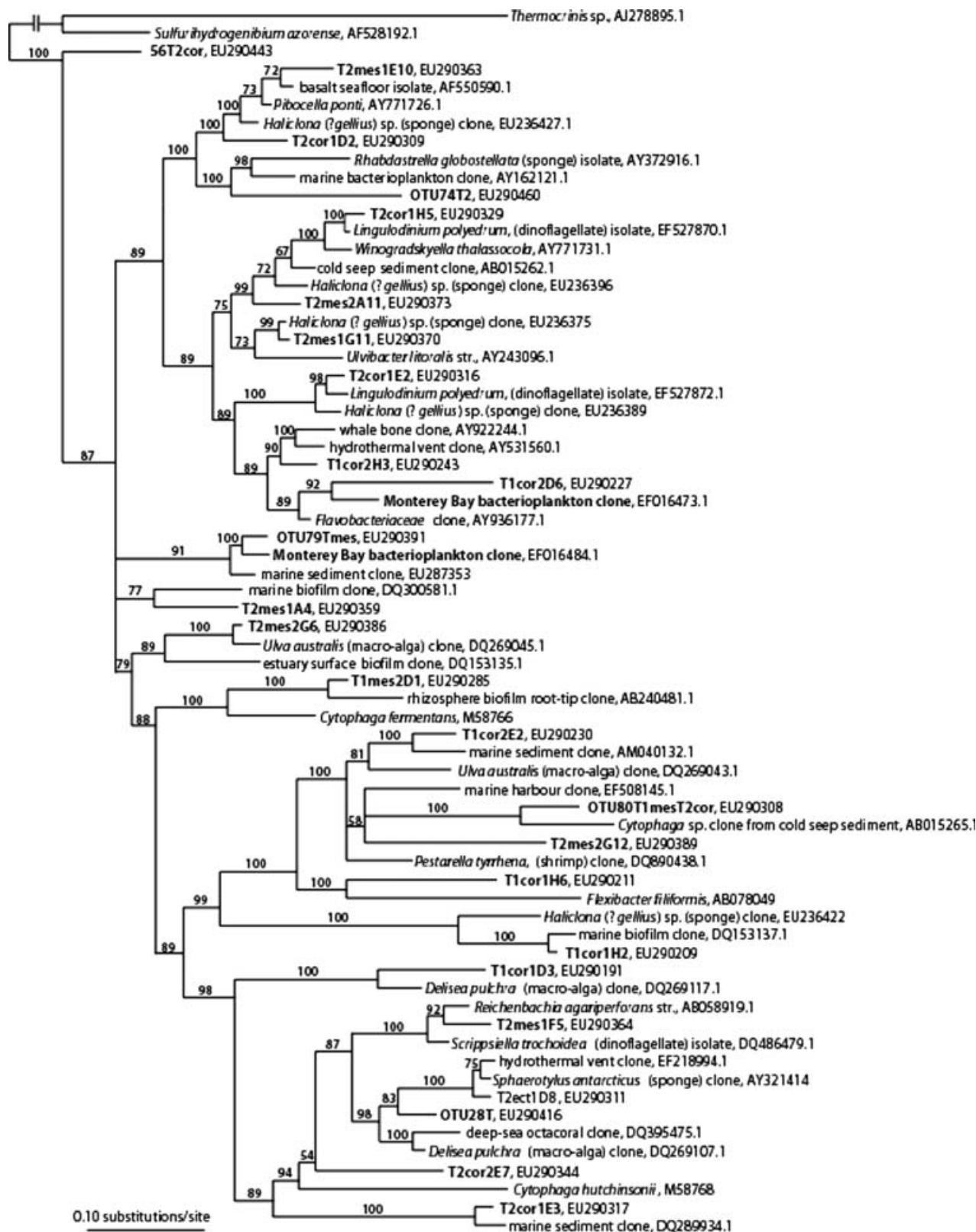

sponges as a source to discover 'new' microorganisms. Sponges from under-represented locations with regard to 16S rRNA gene libraries (Taylor et al. 2007), such as $T$. californiana, may be particularly rich in previously undetected microbes.

\section{Distribution of OTUs in T. californiana tissue}

Overlap of OTUs in tissue samples from different specimens was apparent, but similarity between cortex and mesohyl samples from the same specimen was even more pronounced (Fig. 7). Only six OTUs (08, 50, 61, 76, 78 and 79) were confined to either mesohyl or cortex (singletons excluded), while 37 OTUs were found in both cortex and mesohyl samples (Fig. 8). OTU08 and OTU79 were discovered to be probably local seawater-derived, while the other OTUs that were confined to either mesohyl or cortex have each near neighbors from various sources (sediment, seawater, marine invertebrates), which does not support spatial specificity in sponge tissue. The singletons represent a large part of microbial diversity in $T$. californiana and by definition they are restricted to only one tissue region and may include spatially selective microorganisms. However, singletons are also restricted to only one of the $T$. californiana specimens, and therefore, they are not included in the analysis for spatial specificity. Pyrosequencing of the samples could yield information whether the singletons are really singletons or whether they represent spatio-selective bacteria. 
Fig. 6 a, b Bayesian phylogram of $16 \mathrm{~S}$ rRNA gene sequences from $T$. californiana and their nearest neighbors (greengenes database August $18,2007)$ belonging to other phyla. Archaeoglobus fulgidus and Methanococcus maripaludis were used as outgroup. For further explanation, see Fig. 3

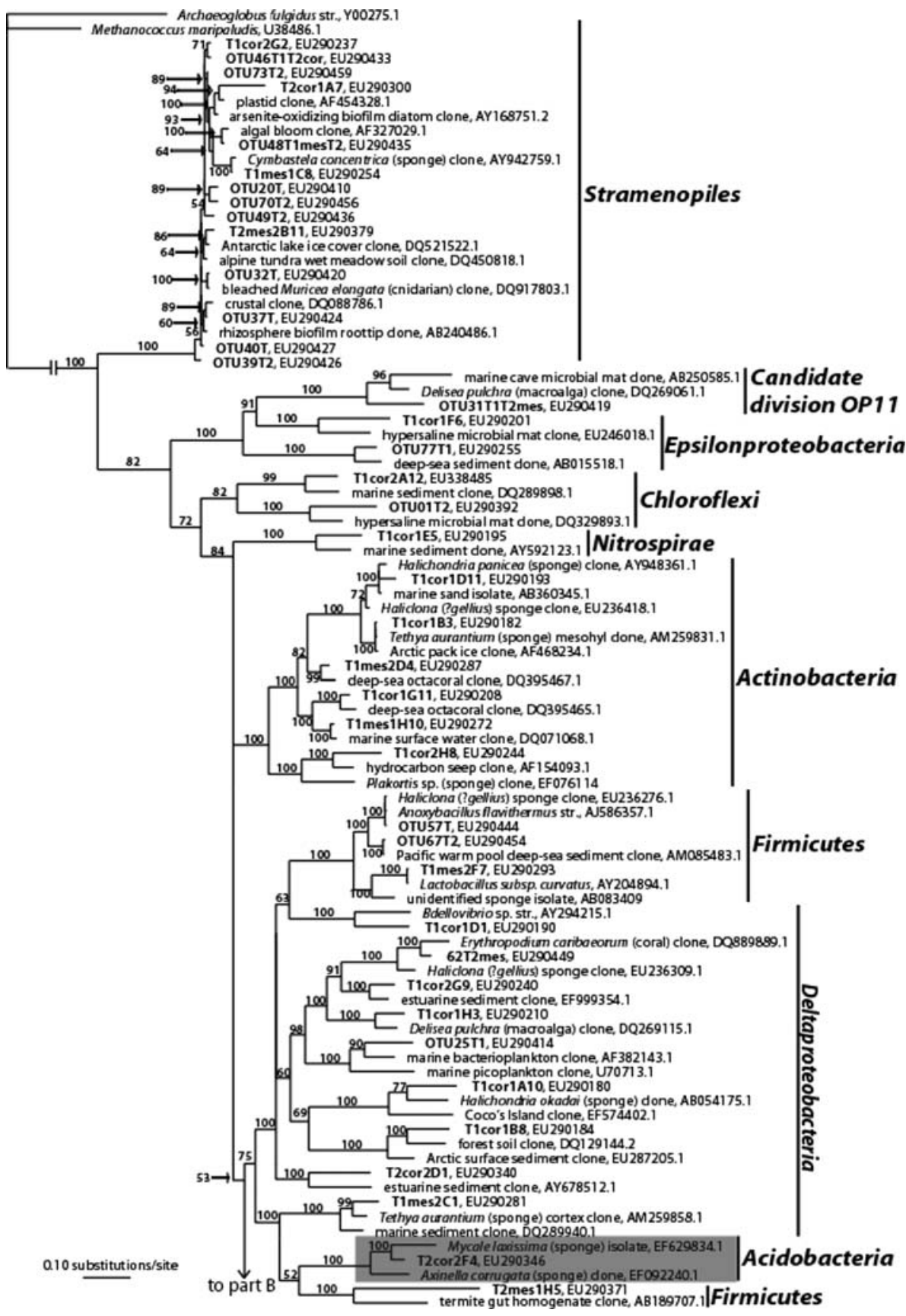

Despite similarities of the microbial communities in $T$. californiana and $T$. aurantium on the phylum-level and, to a lesser extent at the OTU-level, spatio-selectivity of the associated bacteria for T. aurantium could not be confirmed for $T$. californiana. Instead, phylogenetic analysis showed that for a number of cases, clones from mesohyl samples from $T$. californiana shared most identity with cortexderived clones from $T$. aurantium and vice versa (Fig. 4: T2mes2D12, and Fig. 6a: T1cor1B3, T1mes2C1). For T. aurantium, a big shift of the OTUs was measured for samples in different years. Overlap between samples of 2003 and 2004 showed that only 3 out of 51 OTUs could be recovered from samples from both years (Thiel et al. 2007), which could imply that either microbial populations in the sponge are unstable, or that total microbial diversity was not covered. The authors showed with rarefaction analysis that 46 and $58 \%$ of microbial diversities were covered for the cortex and mesohyl, respectively. The remaining uncovered diversity leaves room to either confirm or reject a conclusion on regional specificity of associated microorganisms. However, the fact that Cyanobacteria-affiliated clones, which represented $27 \%$ of all $T$. aurantium clone sequences, were found almost exclusively in the mesohyl suggests that for the Cyanobacteria, spatial selectivity exists in the 
Fig. 6 continued

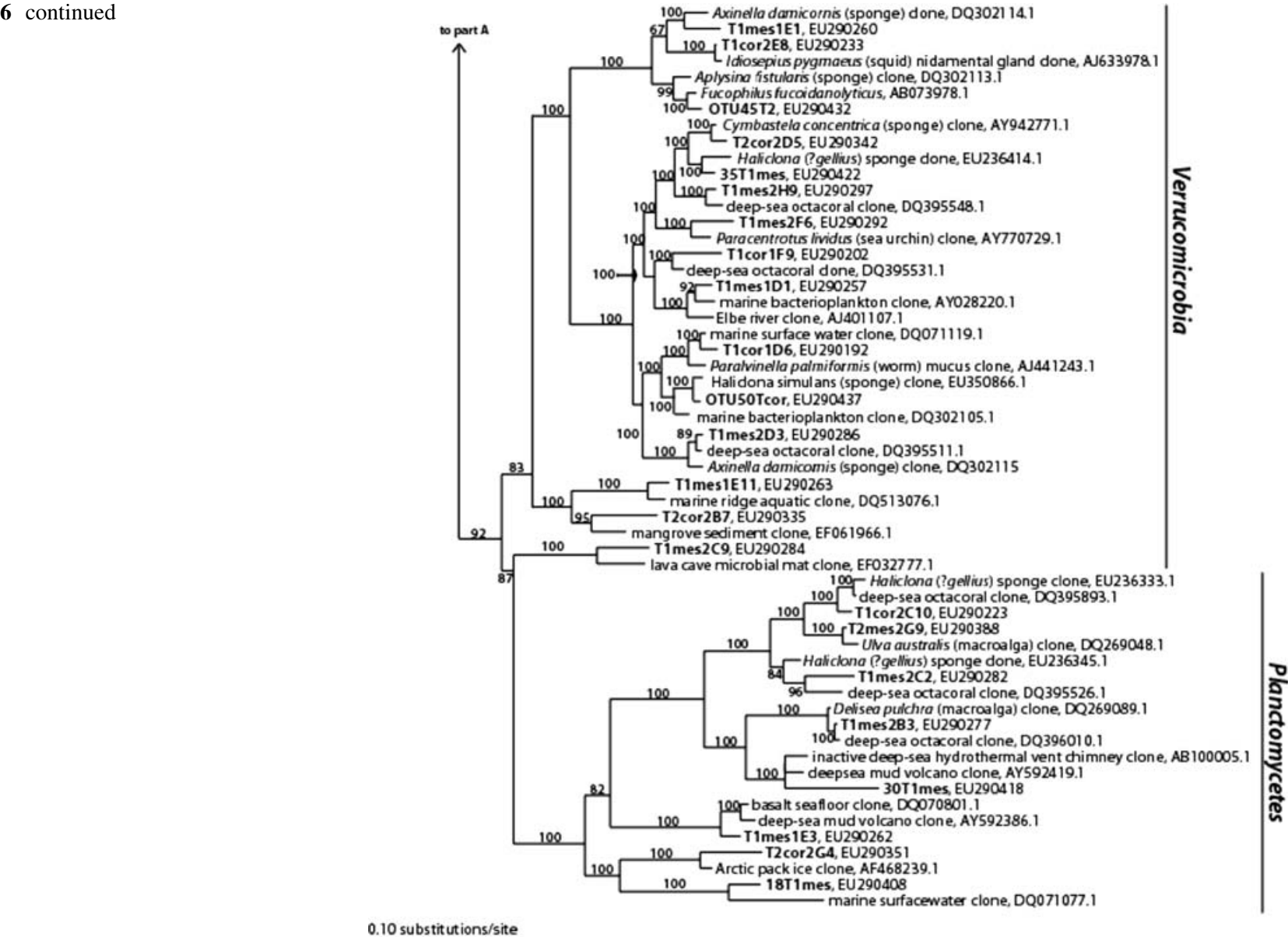

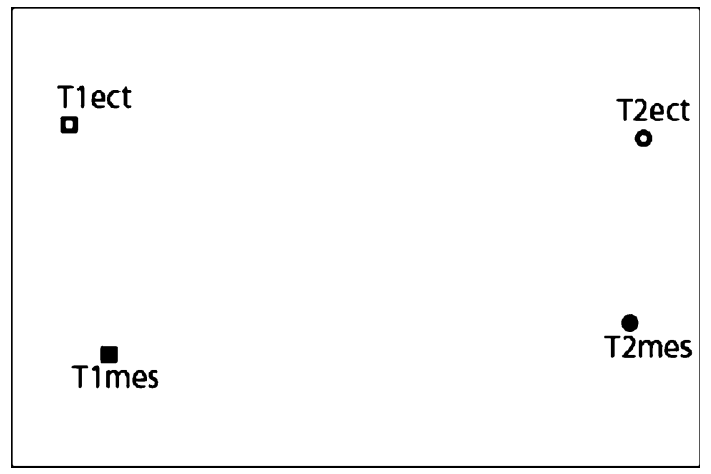

Fig. 7 Bray Curtis analysis of similarity between samples based on OTUs (singletons were not taken into account). The relative distance between the data points is a measure for similarity, i.e., data points that are closer to each other indicate a higher similarity

Mediterranean sponge species. Another study on spatial distribution of bacteria and archaea in the deep-water sponge Polymastia cf. corticata also suggested spatial selectivity of the microorganisms (Meyer and Kuever, 2008). The authors found that Acidobacteria, Actinobacteria and Deltaproteobacteria were sponge- or species- specific and restricted to the mesohyl. The same was true for sponge-specific archaeal sulfate reducers. This result was not confirmed for $T$. californiana, as we did not find spongespecific members of Deltaproteobacteria and Actinobacteria (archaea were not tested), while the only sponge-specific member of the Acidobacteria was detected in an cortex sample. In addition, other sponge-specific OTUs in T. californiana were not restricted to either cortex or mesohyl.

Differences between mesohyl and cortex in T. californiana may exist with respect to the microbial concentration in the different regions, as associated microorganisms are generally more abundant in the mesohyl than in the cortex, which was confirmed for T. aurantium (Thiel et al. 2007). The most pronounced (qualitative) difference we found between cortex and mesohyl samples for $T$. californiana was the number of OTUs detected: $92.5 \pm 9.2$ and $67 \pm 8.5$ OTUs, respectively. However, this may be an artifact caused by different bacterial densities in mesohyl and cortex samples (if they resemble the concentrations in T. aurantium). If the bacterial density is much higher in the latter samples, it would lead to a relative over sampling of the cortex samples compared to the mesohyl samples, because a similar number of clones were used for both 


\section{T. californiana1 T. californiana1\&2 T. californiana2}

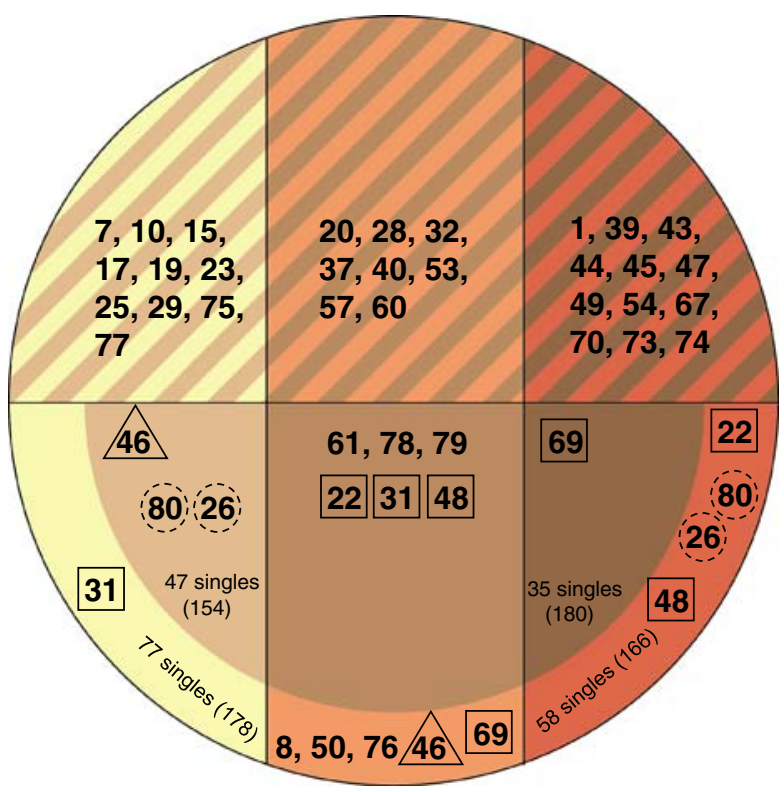

Fig. 8 The distribution of associated microbial species based on OTUs in $T$. californiana tissue samples. Each number refers to the accompanying OTU. The left part of the figure represents $T$. californiana specimen 1, the right part Tethya californiana specimen 2 and the center overlap between the specimens. The upper half represents OTUs that were detected among cortex AND mesohyl samples, and in the lower half OTUs are depicted that were restricted to one of the zones in the sponge. In addition, OTUs that were present in 2 or 3 of the 4 samples (but in a nonsystematic way) are also depicted in the lower half surrounded by squares, triangles and circles. For example, OTU22 was detected in the cortex of T2 and in the mesohyl of $\mathrm{T} 1$ and $\mathrm{T} 2$

regions. This could lead to the isolation of relatively more rare or 'accidental' species from the low-density sample (Gotelli and Colwell 2001). This phenomenon might interfere with rarefaction analysis as it has been noted that if many OTUs of low abundance are present in the data set (as is the case especially for the cortex samples), rarefaction curves tend to converge to a line without an apparent limit and not accurately describe the predicted number of OTUs (Thiel et al. 2007). Therefore, based on qualitative data on microbial diversity and distribution of the diversity in the sponge tissue, we do not observe spatial selectivity of $T$. californiana associates.

Acknowledgments This work was supported by a Marie Curie grant from the European Union (MOIF-CT-2005-022164). The authors wish to thank Mikel Becerro for his help with the statistical analysis.

Open Access This article is distributed under the terms of the Creative Commons Attribution Noncommercial License which permits any noncommercial use, distribution, and reproduction in any medium, provided the original author(s) and source are credited.

\section{References}

Bayer K, Schmitt S, Hentschel U (2008) Physiology, phylogeny and in situ evidence for bacterial and archaeal nitrifiers in the marine sponge Aplysina aerophoba. Environ Microbiol 10:2942-2955

Becerro MA, Lopez NI, Turon X, Uriz MJ (1994) Antimicrobial activity and surface bacterial film in marine sponges. J Exp Mar Biol Ecol 179:195-205

Brümmer F, Pfannkuchen M, Baltz A, Hauser T, Thiel V (2008) Light inside sponges. J Exp Mar Biol Ecol 367:61-64

Castresana J (2000) Selection of conserved blocks from multiple alignments for their use in phylogenetic analysis. Mol Biol Evol 17:540-552

Chelossi E, Milanese M, Milano A, Pronzato R, Riccardi G (2004) Characterisation and antimicrobial activity of epibiotic bacteria from Petrosia ficiformis. J Exp Mar Biol Ecol 309:21-33

Clarke KR, Warwick RM (2001) Changes in marine communities: an approach to statistical analysis and interpretation, 2nd edn. PRIMER-E, Plymouth, p 172

Faulkner DJ (2000) Highlights of marine natural products chemistry (1972-1999). Nat Prod Rep 17:1-6

Felske A, Rheims H, Woltherink A, Stackebrandt E, Akkermans ADL (1997) Ribosome analysis reveals prominent activity of an uncultured member of the class Actinobacteria in grassland soils. Microbiology 143:2983-2989

Friedrich AB, Fischer I, Proksch P, Hacker J, Hentschel U (2001) Temporal variation of the microbial community associated with the mediterranean sponge Aplysina aerophoba. FEMS Microbiol Ecol 38:105-113

Gaino E, Sará M (1994) Siliceous spicules of Tethya seychellensis (Porifera) support the growth of a green alga-a possible light conducting system. Mar Ecol Prog Ser 108:147-151

Gotelli NJ, Colwell RK (2001) Quantifying biodiversity: procedures and pitfalls in the measurement and comparison of species richness. Ecol Lett 4:379-391

Grant AJ, Trautman DA, Menz I, Hinde R (2006) Separation of two cell signalling molecules from a symbiotic sponge that modify algal carbon metabolism. Biochem Biophys Res Commun 348:92-98

Hallam SJ, Konstantinidis KT, Putnam N, Schleper C, Watanabe Y, Sugahara J, Preston C, De la Torre J, Richardson PM, DeLong EF (2006) Genomic analysis of the uncultivated marine crenarchaeote Cenarchaeum symbiosum. Proc Natl Acad Sci 103:18296-18301

Hentschel U, Hopke J, Horn M, Friedrich AB, Wagner M, Hacker J, Moore BS (2002) Molecular evidence for a uniform microbial community in sponges from different oceans. Appl Environ Microbiol 68:4431-4440

Hentschel U, Fieseler L, Wehrl M, Gernert C, Steinert M, Hacker J, Horn M (2003) Microbial diversity of marine sponges. In: Müller WEG (ed) Marine molecular biotechnology. Springer, Berlin, pp 59-88

Hill M, Hill A, Lopez N, Harriott O (2006) Sponge-specific bacterial symbionts in the Caribbean sponge, Chondrila nucula (Demospongia, Chondrosida). Mar Biol 148:1221-1230

Hoffmann F, Larsen O, Thiel V, Rapp HT, Pape T, Michaelis W, Reitner J (2005) An anaerobic world in sponges. Geomicrobiol J 22:1-10

Hoffmann F, Røy H, Bayer K, Hentschel U, Pfannkuchen M, Brümmer F, De Beer D (2008) Oxygen dynamics and transport in the Mediterranean sponge Aplysina aerophoba. Mar Biol 153:1257-1264

Hoffmann F, Radax R, Woebken D, Holtappels M, Lavik G, Rapp HT, Schläppy ML, Schleper C, Kuypers MMM (2009) Complex nitrogen cycling in the sponge Geodia barretti. Environ Microbiol. doi:10.1111/j.1462-2920.2009.01944.x 
Huber T, Faulkner G, Hugenholtz P (2004) Bellerophon: a program to detect chimeric sequences in multiple sequence alignments. Bioinformatics 20:2317-2319

Huelsenbeck JP, Ronquist F (2001) MRBAYES: Bayesian inference of phylogenetic trees. Bioinformatics 17:754-755

Hurlbert S (1971) The nonconcept of species diversity: a critique and alternative parameters. Ecology 52:577-586

Kennedy J, Codling CE, Jones BV, Dobson ADW, Marchesi JR (2008) Diversity of microbes associated with the marine sponge, Haliclona simulans, isolated from Irish waters and identification of polyketide synthase genes from the sponge metagenome. Environ Microbiol 10:1888-1902

Lane DJ (1991) 16S/23S rRNA sequencing. In: Stackebrandt E, Goodfellows $M$ (eds) Nucleic acid techniques in bacterial systematics. John Wiley, New York, pp 115-175

Lee YK, Lee JH, Lee HK (2001) Microbial symbiosis in marine sponges. J Microbiol 39:254-264

Ludwig W, Strunk O, Westram R, Richter L, Meier H, Yadhukumar, Buchner A, Lai T, Steppi S, Jobb G, Forster W, Brettske I, Gerber S, Ginhart AW, Gross O, Grumann S, Hermann S, Jost R, Konig A, Liss T, Lussmann R, May M, Nonhoff B, Reichel B, Strehlow R, Stamatakis A, Stuckmann N, Vilbig A, Lenke M, Ludwig T, Bode A, Schleifer KH (2004) ARB: a software environment for sequence data. Nucleic Acids Res 32:1363-1371

Massana R, Castresana J, Balagué V, Guillou L, Romari K, Groisillier A, Valentin K, Pedrós-Alió C (2004) Phylogenetic and ecological analysis of novel marine Stramenopiles. Appl Environ Microbiol 70:3528-3534

Meyer B, Kuever J (2008) Phylogenetic diversity and spatial distribution of the microbial community associated with the Caribbean deep-water sponge Polymastia cf corticata by $16 \mathrm{~S}$ rRNA, aprA, and amoA gene analysis. Microb Ecol 56:306-321

Müller WEG, Grebenjuk VA, Thakur NL, Thakur AN, Batel R, Krasko A, Müller IM, Breter HJ (2004) Oxygen-controlled bacterial growth in the sponge Suberites domuncula: toward a molecular understanding of the symbiotic relationships between sponge and bacteria. Appl Environ Microbiol 70:2332-2341

Osinga R, Armstrong E, Burgess JG, Hoffmann F, Reitner J, Schumann-Kindel G (2001) Sponge-microbe associations and their importance for sponge bioprocess engineering. Hydrobiologia 461:55-62

Ridley CP, Faulkner DJ, Haygood MG (2005) Investigation of oscillatoria spongeliae-dominated bacterial communities in four Dictyoceratid sponges. Appl Environ Microbiol 71:7366-7375
Ronquist F, Huelsenbeck JP, Van der Mark O (2005) MrBayes 3.1 manual. School of Computational Science, Florida State University, USA

Sipkema D, Holmes B, Nichols SA, Blanch HW (2009) Biological characterization of $H$. (?gellius) sp.: sponge and symbionts. Microb Ecol 58:903-920

Stackebrandt E, Ebers J (2006) Taxonomic parameters revisited: tarnished gold standards. Microbiol Today 33:152-155

Suzuki MT, Béjà O, Taylor LT, DeLong EF (2001) Phylogenetic analysis of ribosomal RNA operons from uncultivated coastal marine bacterioplankton. Environ Microbiol 3:323-331

Suzuki MT, Preston CM, Béjà O, De la Torre JR, Steward GF, DeLong EF (2004) Phylogenetic screening of ribosomal RNA gene-containing clones in bacterial artificial chromosome (BAC) libraries from different depths in Monterey Bay. Microb Ecol 48:473-488

Swofford DL (2003) Phylogenetic analysis using parsimony ( ${ }^{*}$ and other methods). Version 4. Sinauer Associates, Sunderland

Taylor MW, Schupp PJ, Dahllöf I, Kjelleberg S, Steinberg PD (2004) Host specificity in marine sponge-associated bacteria and potential implications for marine microbial diversity. Environ Microbiol 6:121-130

Taylor MW, Radax R, Steger D, Wagner M (2007) Sponge-associated microorganisms: evolution, ecology and biotechnological potential. Microbiol Mol Biol Rev 71:295-347

Thiel V, Neulinger SC, Staufenberger T, Schmaljohann R, Imhoff JF (2007) Spatial distribution of sponge-associated bacteria in the Mediterranean sponge Tethya aurantium. FEMS Microbiol Ecol 59:47-63

Wichels A, Würtz S, Döpke H, Schütt C, Gerdts G (2006) Bacterial diversity in the breadcrumb sponge Halichondria panicea (Pallas). FEMS Microbiol Ecol 56:102-118

Wilkinson CR (1980) Nutrient translocation from green algal symbionts to the freshwater sponge Ephydatia fluviatilis. Hydrobiologia 75:241-250

Wilkinson CR, Garrone R (1980) Nutrition of marine sponges. Involvement of symbiotic bacteria in the uptake of dissolved carbon. In: Smith DC, Tiffon Y (eds) Nutrition in the lower metazoa. Pergamon, pp 157-161

Zwickl DJ (2006) Genetic algorithm approaches for the phylogenetic analysis of large biological sequence datasets under the maximum likelihood criterion. Ph.D. dissertation, UT Austin, USA 